\section{A proposed system for screening tooth wear}

\author{
D. Bartlett ${ }^{1}$
}

IN BRIEF
- Presents a convenient and simple way to
record tooth wear in practice.
- The four levels in the proposed system
can be easily understood.
- Simple associated treatment options give
additional help.

This paper presents a useful and convenient way to record the severity of tooth wear for patients seen in primary care. The index developed in collaboration with colleagues in Europe because there is widespread belief that tooth wear and in particular erosion is a growing issue for practitioners. The index is based on the BPE and uses similar protocols. It is designed to be simple to use, easily recorded in the notes, and gives practitioners the opportunity to record that the wear has been examined and considered. The four level index uses 0 (no wear), 1 (initial loss of surface texture), 2 ( $<50 \%$ loss of surface) and 3 ( $>50 \%$ loss of surface) to record the severity of wear.

\section{RECORDING EROSION AND TOOTH WEAR IN PRIMARY CARE}

The management of tooth wear in primary care often presents challenges. Data from prevalence studies in adults, ${ }^{1}$ adolescents ${ }^{2,3}$ and children ${ }^{4}$ indicate that it is a common clinical finding and as a recent systematic review suggests, the incidence and severity increases with age. ${ }^{5}$ While our understanding of the aetiology and pathogenesis of tooth wear is developing it is not at the same level of knowledge as other comparable dental conditions such as caries and periodontal disease. Admittedly, research into tooth wear, particularly erosion, has grown significantly over recent years with many more countries appreciating the impact of tooth wear on their populations. However, the gaps in our understanding and the clinical dilemma of 'what to do' seem to be frequent concerns for many practitioners.

The terminology used with tooth wear has not received universal acceptance. There are terms such as 'tooth surface loss' and 'tooth wear' which are understood by

'Department of Prosthodontics, King's College London Dental Institute, Floor 25, Guy's Tower, London Bridge, SE1 9RT

Correspondence to: Professor David Bartlett

Email: david.bartlett@kcl.ac.uk

\section{Refereed Paper}

Accepted 1 February 2010

DOI: 10.1038/sj.bdj.2010.205

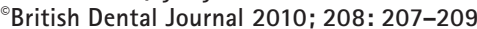

many to mean identical clinical findings. But some researchers and practitioners have slightly different interpretations. ${ }^{6}$ The same situation occurs with erosion and tooth wear. Damage to teeth caused by acids is called erosion but in most situations other forms of tooth wear occur at the same time. Therefore, using the term 'erosion' to describe changes to teeth is almost always incorrect. However, many European countries prefer the term 'erosion' to describe non-cariogenic changes to teeth and others consider parafunctional activity to be always attrition. The situation is confusing. The term 'tooth wear' is generally understood by patients and a preferred term of many practitioners and in used in this paper.

The role of acidic diets and gastric acid in erosive tooth wear is acknowledged and reasonably well understood. ${ }^{7}$ The strength of the dietary acids is an important risk factor, particularly those with a high titratable acidity such as citrus-based fruits and drinks. ${ }^{7}$ But the frequency of consumption of acidic foods or drinks, particularly snacking, is acknowledged to be the greatest the risk of developing erosive tooth wear. ${ }^{7,8}$ Preventive advice is based around avoiding snacking on acidic foods and drinks, which is the same message given for caries. Frequently drinking carbonated water is unlikely to cause erosion but if the drink was more acidic, particularly with a citrus base, the risk of developing erosion is much higher. Therefore the advice needs to be clear and pragmatic.

The most common cause of tooth wear is probably dietary acids but the most severe forms are likely to be related to gastric causes. ${ }^{9}$ The identification of erosive tooth wear caused by gastric acid, either a result of gastro-oesophageal reflux or of an eating disorder, is more challenging in primary care but an underlying history of regurgitation or vomiting of gastric acids into the mouth may provide some help in the diagnosis. Although medical tests are possible, and have been shown to have value in defining a diagnosis of gastric cause ${ }^{10}$ they are often unrealistic for GDPs to arrange with their medical colleagues. Consequently most have been conducted within hospital environments. The medical management of these cases must be coordinated by the general medical practitioner (GMP) and it may be helpful to raise concern about patients who are suspected of suffering from undiagnosed gastric causes of tooth wear.

There is increasing support for the view that once tooth wear has been diagnosed the progression is slow. ${ }^{11,12}$ These studies imply that dietary advice reduces the rate of tooth wear and eliminates the need for clinical intervention. Currently work from our research group supports the conclusion that tooth wear is a slow process once preventive advice has been successfully implemented. ${ }^{11-13}$ Therefore, with these 
Table 1 Clinical sequence when using the BEWE

1. Diagnose the presence of tooth wear; eliminate teeth with trauma and developmental defects from the score

2. Examine all teeth and all surfaces of teeth in the mouth for tooth wear

3. Identify in each quadrant the most severely affected tooth with wear

4. Conduct BEWE score.

\begin{tabular}{|c|c|}
\hline Score & Features \\
\hline 0 & No erosive tooth wear \\
\hline 1 & Initial loss of surface texture \\
\hline 2 & $\begin{array}{l}\text { Distinct defect, hard tissue loss }<50 \% \text { of } \\
\text { the surface area }\end{array}$ \\
\hline 3 & Hard tissue loss $\geq 50 \%$ of the surface area \\
\hline
\end{tabular}

findings in mind tooth wear probably progress slowly over a lifetime but in some cases short bursts of activity may produce more severe wear but which may be then followed by longer periods of inactivity. These findings on progression are no different to that for caries and periodontal disease, both of which have been found to be preventable although there is a continuing at-risk susceptibility. However, individual variations occur and it is difficult to predict on an individual basis.

Many dental hospitals/secondary care settings receive referrals on the management of tooth wear and so get a somewhat skewed vision of what is happening in primary care. If a practitioner has a stable pool of patients it is possible that they will see the development and progression of tooth wear even if this occurs during short time periods of time. But many practitioners and patients move between practices making long term monitoring of tooth wear, in terms of 5-10 years, challenging. Therefore, most practitioners examine patients with tooth wear for the first time at various stages in the development of the condition. It is tempting in these situations to be worried and think that restorative intervention is needed because of the concern that if nothing is done the condition may worsen, leading to concerns about supervised neglect. The evidence does not support this fear as tooth wear, once diagnosed, normally slows and rarely leads to tooth destruction. Therefore, a simple and

\section{Table 3 Complexity levels as a guide to clinical management ${ }^{15}$}

\begin{tabular}{|c|c|c|}
\hline $\begin{array}{l}\text { Complexity } \\
\text { level }\end{array}$ & $\begin{array}{l}\text { Cumulative score } \\
\text { of all sextants }\end{array}$ & Management \\
\hline 0 & $\begin{array}{l}\text { Less than } \\
\text { or equal to } 2\end{array}$ & $\begin{array}{l}\text { Routine maintenance and observation } \\
\text { Repeat at 3-year intervals }\end{array}$ \\
\hline 1 & Between 3 and 8 & $\begin{array}{l}\text { Oral hygiene and dietary assessment, and advice, routine maintenance } \\
\text { and observation } \\
\text { Repeat at 2-year intervals }\end{array}$ \\
\hline 2 & Between 9 and 13 & $\begin{array}{l}\text { Oral hygiene and dietary assessment, and advice, identify the main } \\
\text { aetiological factor(s) for tissue loss and develop strategies to eliminate } \\
\text { respective impacts } \\
\text { Consider fluoridation measures or other strategies to increase the } \\
\text { resistance of tooth surfaces } \\
\text { Ideally, avoid the placement of restorations and monitor erosive wear } \\
\text { with study casts, photographs, or silicone impressions } \\
\text { Repeat at 6-12 month intervals }\end{array}$ \\
\hline 3 & 14 and over & $\begin{array}{l}\text { Oral hygiene and dietary assessment, and advice, identify the main } \\
\text { aetiological factor(s) for tissue loss and develop strategies to eliminate } \\
\text { respective impacts } \\
\text { Consider fluoridation measures or other strategies to increase the } \\
\text { resistance of tooth surfaces } \\
\text { Ideally, avoid restorations and monitor tooth wear with study casts, } \\
\text { photographs, or silicone impressions } \\
\text { Especially in cases of severe progression consider special care that may } \\
\text { involve restorations } \\
\text { Repeat at 6-12 month intervals }\end{array}$ \\
\hline
\end{tabular}

easy screening tool would allow GDPs to assess the severity of tooth wear and record this information in the notes.

The development and adoption of the BPE (basic periodontal examination) is an ideal example to follow for the management of tooth wear. ${ }^{14}$ When the screening tool was first proposed the level of clinical knowledge and understanding of practitioners was probably at a comparable stage to that of tooth wear today. Research had identified risk factors but the level of understanding by many practitioners and their ability to scrutinise and screen patients was underdeveloped. The BPE provided them with a simple but effective tool to use in everyday practice and which in a very convenient way recorded the level of disease in the notes. This started to protect practitioners against claims of supervised neglect since it showed that an assessment had occurred. In such a similar way tooth wear needs this screening and assessment tool for GDPs to use in their clinical practice.

Recently a screening tool, devised by a group of clinicians from the UK, Switzerland and Germany, was proposed and is shown in Tables 1, 2 and $3 .{ }^{15}$ The tool, called the BEWE (basic erosive wear examination), was devised using many of the concepts first proposed by those clinicians involved with the BPE and aims to screen patients for tooth wear. Firstly, clinicians identify the tooth with the most severe tooth wear in a quadrant and unlike the BPE all teeth and all surfaces are examined (Figs 1 and 2). It requires a basic level of knowledge and skills by a practitioner in diagnosing tooth wear, otherwise known as tooth surface loss, and does not record change to teeth as a result of trauma or developmental defects. Once the tooth with the most severe wear is identified the severity is recorded using a simple four level scheme. Zero signifies the absence of tooth wear or a normal appearance; 1 indicates initial loss of surface detail; 2 is exposure of wear involving less than 50\% of the surface and 3 involves greater than 50\% loss of the surface. Although dentine exposure can be a useful indicator to the severity of tooth wear it can be confusing if the colour is difficult to distinguish from that of enamel. The scoring scheme is repeated in all quadrants to give a cumulative score from a minimum score of 0 to a maximum score of 18 . The results are recorded in the notes using a similar style of box as for the BPE. It gives the practitioner a base-line and simple method to record severity of tooth wear. The cumulative score is matched to a list of interventions which can assist in the management of the condition.

The BEWE is a simple scoring system which gives an indication of the severity of tooth wear. In those cases where the tooth wear is more severe a more detailed record is needed in the notes. Like the BPE 


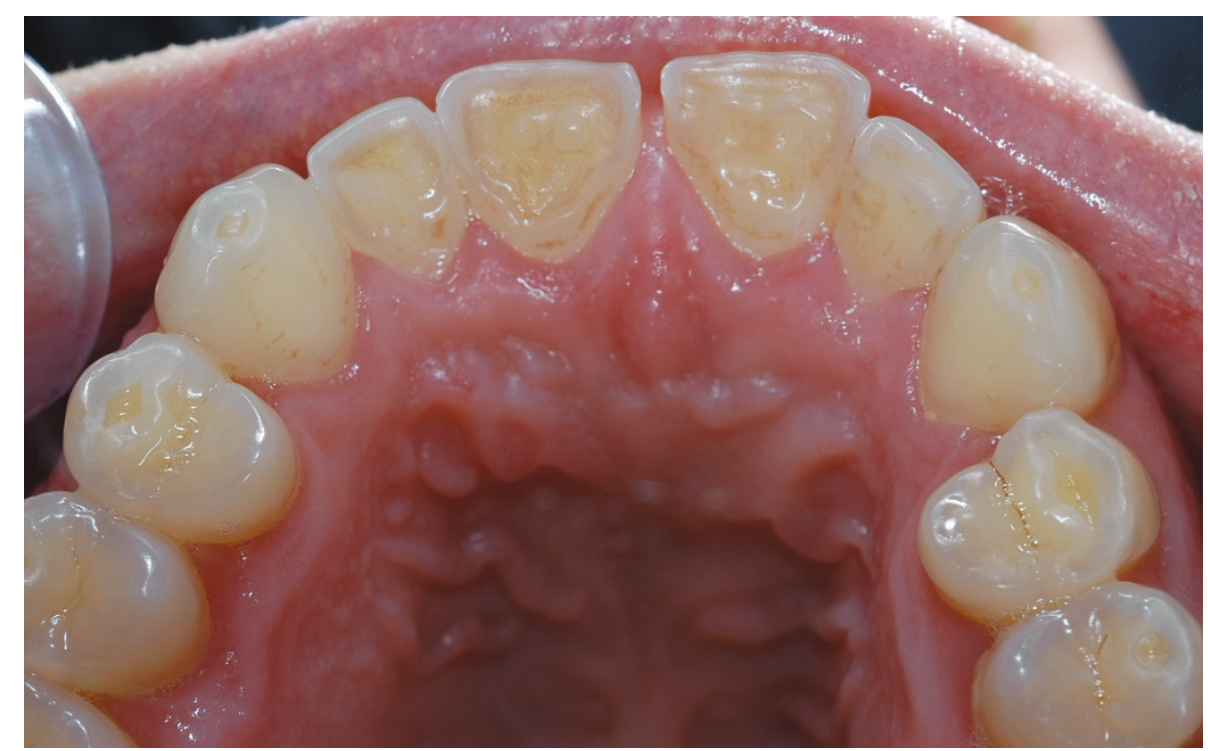

Fig. 1 This image shows worn teeth in the maxilla. The wear has penetrated dentine on the palatal surfaces of the central incisors. The wear on the premolars is less severe but there is dentine exposed on both sides but there are also enamel facets on the palatal cusps. A BEWE score on the upper anterior quadrant would be 3 and on the posterior quadrants a score on the imageís left and right would be 2

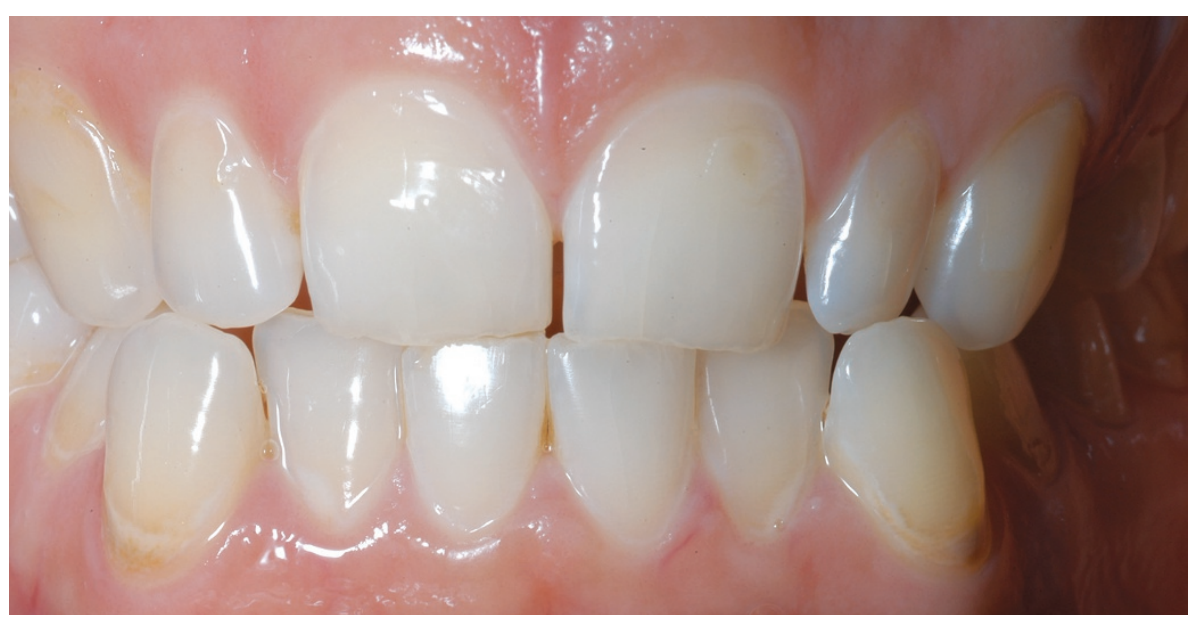

Fig. 2 The most severely worn tooth in the upper anterior quadrant are the lateral incisors with less than $50 \%$ of the surface involved and the BEWE score would be 2 . The same score would be given to the lower anterior quadrant. From what is visible on the right side the level of wear on the premolars and molar would also be less than $50 \%$ of the surface and so another score of 2 would be given. If the pattern was repeated on all six quadrants the cumulative score would be 8 indicating level 2 and the management would focus around prevention

the higher levels indicating greater severity need further evaluation and treatment. The scoring system is sufficiently flexible to overcome areas of wear which are localised. For example, for patients with palatal tooth wear where the severity is limited to the upper anterior region. In the author's clinical experience there will be some evidence of wear on other teeth; it may not be as extensive but multiple recordings of less than $50 \%$ loss of surface would be seen.

The distinction between the cumulative scores is arbitrary. They are not based on research but on clinical experience of the authors of the original article. ${ }^{15}$
Consequently the divisions may vary between national boundaries around the world and will to some extent need careful evaluation. However, complexity level 1 and 2 represent low risk factors and tooth wear levels which are unlikely to affect the prognosis of the teeth. The dietary advice and review structure is based on this assumption. However, as the complexity level increases to 3 or 4 the impact of tooth wear is more important on the management. As the severity of wear increases the timing of monitoring becomes more frequent and the possibility of restorations develops.
The intention of the BEWE is not to measure progression, as the distinction between the various levels is crude. Also, different practitioners will interpret the severity of wear differently and provide different scores. The value of using the BEWE is that once conducted it records that tooth wear has been examined and encourages a preventive approach to limit progression. As the scoring system becomes more commonly adopted it will undergo various changes which will improve its use. The benefit of using a simple scheme is that it can be adapted more easily than a more complex one. The management of tooth wear, in common with many parts of restorative dentistry, is open to personal interpretation and financial consideration but the major advantage is that it records in the patients notes that the tooth wear has been examined and diagnosed.

1. Fares J, Chiu K, Ahmad N, Shirodaria S, Bartlett D W. A new index of tooth wear. Reproducibility and application to a sample of 18-30 year old university students. Caries Res 2009; 43: 119-125.

2. Bardsley P F, Taylor S, Milosevic A. Epidemiological studies of tooth wear and dental erosion in 14-year-old children in North West England. Part 1 The relationship with water fluoridation and social deprivation. Br Dent J 2004; 197: 413-416.

3. Bartlett D W, Coward PY, Nikkah C, Wilson R F. The prevalence of tooth wear in a cluster sample of adolescent schoolchildren and its relationship with potential explanatory factors. Br Dent J 1998; 184: 125-129.

4. O'Brien M. Children's dental health in the United Kingdom 1993. pp 1-130. London: OPCS, 1993.

5. Van 't Spijker A, Rodrigues J M, Kreulen C M et al. Prevalence of tooth wear in adults. Int J Prosthodont 2009; 22: 35-42.

6. Bartlett D W, Phillips K M, Smith B G N. A difference of perspective - the North American and European interpretations of tooth wear. Int J Prosthodont 1999; 12: 401-408.

7. Lussi A, Jaeggi T, Zero D. The role of diet in the aetiology of dental erosion. Caries Res 2004; 38(Suppl 1): 34-44.

8. Moazzez R, Smith B G N, Bartlett D W. Oral pH and drinking habit during ingestion of a carbonated drink in a group of adolescents with dental erosion. J Dent 2000; 28: 395-397.

9. Bartlett D W. The role of erosion in tooth wear: aetiology, prevention and management. Int Dent J 2005; 55(4 Suppl 1): 277-284.

10. Bartlett D W, Evans D F, Anggiansah A, Smith B G N. A study of the association between gastrooesophageal reflux and palatal dental erosion. Br Dent J 1996; 181: 125-131.

11. Bartlett $D$ W. Retrospective long term monitoring of tooth wear using study models. Br Dent J 2003; 194: 211-213.

12. Bartlett D W, Palmer I, Shah P. An audit of study casts used to monitor tooth wear in general practice. Br Dent J 2005; 199: 143-145.

13. Sundaram $G$, Watson $T$, Bartlett D. Clinical measurement of palatal tooth wear following coating by a resin sealing system. Oper Dent 2007; 32: 539-543.

14. Smales F C, Mosedale R F, Floyd P M. Policy for periodontal care. Br Dent J 1987; 163: 167-169.

15. Bartlett D, Ganss C, Lussi A. Basic Erosive Wear Examination (BEWE): a new scoring system for scientific and clinical needs. Clin Oral Investig 2008; 12: 65-68. 